\title{
HOT ELECTRON ENERGY DISTRIBUTIONS FROM \\ ULTRA-INTENSE LASER SOLID INTERACTIONS
}

H. Chen, S. C. Wilks, W. L. Kruer, S. Moon, N. Patel, P. K. Patel, R. Shepherd, R. Snavely

December 9, 2005

The Fourth International Conference on Inertial Fusion Sciences and Applications

Biarritz, France

September 4, 2005 through September 9, 2005 
This document was prepared as an account of work sponsored by an agency of the United States Government. Neither the United States Government nor the University of California nor any of their employees, makes any warranty, express or implied, or assumes any legal liability or responsibility for the accuracy, completeness, or usefulness of any information, apparatus, product, or process disclosed, or represents that its use would not infringe privately owned rights. Reference herein to any specific commercial product, process, or service by trade name, trademark, manufacturer, or otherwise, does not necessarily constitute or imply its endorsement, recommendation, or favoring by the United States Government or the University of California. The views and opinions of authors expressed herein do not necessarily state or reflect those of the United States Government or the University of California, and shall not be used for advertising or product endorsement purposes. 
The headers will be insert by the Publisher The headers will be insert by the Publisher The headers will be insert by the Publisher

\title{
HOT ELECTRON ENERGY DISTRIBUTIONS FROM ULTRA- INTENSE LASER SOLID INTERACTIONS
}

\author{
Hui Chen, S. C. Wilks, W. L. Kruer, S. Moon, N. Patel, P. K. Patel, R. Shepherd and R. \\ Snavely
}

Lawrence Livermore National Laboratory, Livermore, CA 94550, USA

\begin{abstract}
We present experimental data of electron energy distributions from ultra-intense $\left(>10^{19} \mathrm{~W} / \mathrm{cm}^{2}\right)$ laser-solid interactions using the Rutherford Appleton Laboratory Vulcan petawatt laser. These measurements were made using a CCD-based magnetic spectrometer. We present details on the distinct effective temperatures that were obtained for a wide variety of targets as a function of laser intensity. It is found that as the intensity increases from $10^{17} \mathrm{~W} / \mathrm{cm}^{2}$ to $10^{19} \mathrm{~W} / \mathrm{cm}^{2}$, a 0.4 dependence on the laser intensity is found. Between $10^{19} \mathrm{~W} / \mathrm{cm}^{2}$ and $10^{20} \mathrm{~W} / \mathrm{cm}^{2}$, a gradual rolling off of temperature with intensity is observed.
\end{abstract}

\section{INTRODUCTION.}

Hot electron generation and transport from ultra-intense laser solid interactions is fundemental to the understanding of high energy density physics. One useful metric to quantify the hot electrons generated during these interactions is the hot electron energy distribution, hereafter referred to as the effective electron temperature, $\mathrm{T}_{\text {hot }}$. Although it is generally agreed that, as shown in previous studies (see review by Gibbon and Forster [1]), higher laser intensity results higher electron temperature, and at ultrahigh intensities $\left(\mathrm{I}^{2}>10^{18} \mathrm{Wcm}^{-2} \mu \mathrm{m}^{2}\right)$ electrons are strongly relativistic with effective temperatures that scale with the ponderomotive potential of the laser [2], the predictions of the electron temperatures are not unambiguous due to the complicated physics processes involved. In Figure 1, we plot the data presented in Gibbon and Forster [1], along with the predictions of a set of 2D PIC simulations, as well as the ponderomotive scaling [2], which is given by

$$
k T_{\text {hot }} \sim m_{e} c^{2}\left\lfloor\sqrt{1+\frac{I \lambda^{2}}{2.8 \times 10^{18}}}-1\right\rfloor
$$




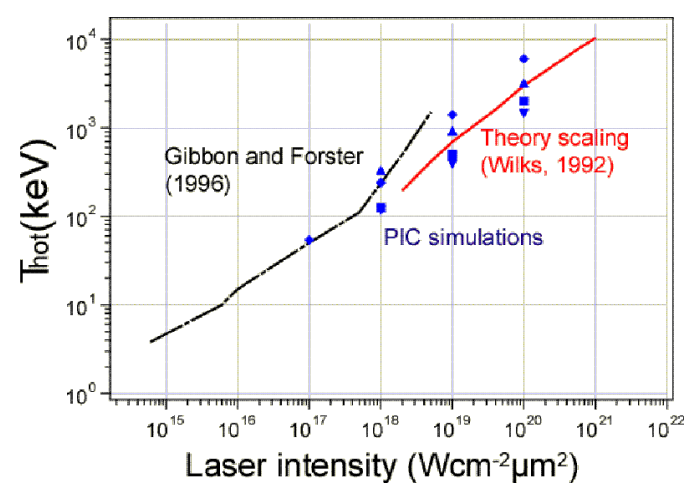

Figue 1. Hot electron temperature from simulation and theoretical scalling. The black was reproduced from the review article by Gibbon and Forster [1], which agreed well with existing experimental data. The discreate points were from our 2D PIC calculations at various conditions (from four simulation conditions: blue triangles for with prepulse at 0 degree incident angle; blue squares for no prepulse at 0 degree incident angle; blue diamonds for with prepulse at 45 degree incident angle and blue upside-down for no prepulse at 45 degree incident angle) and the red line is from theoretical scalling by Wilks et al. [2].

To measure the hot electron distribution from ultra high intense short pulse laser plasma interaction, we used a magnetic electron spectrometer that has been described previously [3]. This instrument was fielded at both the Rutherford Appleton Laboratory (RAL) Vulcan petawatt laser [4] and the LLNL Jupiter laser facility. The results from both experiments are discussed in this paper.

\section{EXPERIMENTAL RESULTS}

We begin by describing the experiments performed on the LLNL Jupiter facility Callisto (previously referred as JanUSP) Ti:sapphire laser. It has a pulse length of $100 \mathrm{fs}$ and delivers up to $10 \mathrm{~J}$ laser energy at $800 \mathrm{~nm}$. The laser is focused with an $\mathrm{f} / 2$ parabola to a focus spot size of about $3.5 \mu \mathrm{m}$, and the laser intensity ranges from $10^{17}-10^{20} \mathrm{Wcm}^{-2}$. The laser is incident on the various target at 22.5 degrees off normal. The spectrometer slit is aligned 30 degrees from the laser beam, i.e. 52.5 degrees off normal, and $23 \mathrm{~cm}$ away from the target.

At lower intensity, we found the hot electron temperature agreed well with PIC simulations and theory [5]. At higher intensity, however, we observed hot electron temperatures approching a 'saturation' level as the laser intensity increase from mid of $10^{19}$ to $10^{20} \mathrm{Wcm}^{-2}$, as shown in Fig. 2. Note that the hot electron temperature is defined as the slope of the exponential decay of the distribution :

$$
T_{h o t}=\frac{E_{1}-E_{2}}{\ln \left(f\left(E_{1}\right) / f\left(E_{2}\right)\right)}
$$

\section{Error!}




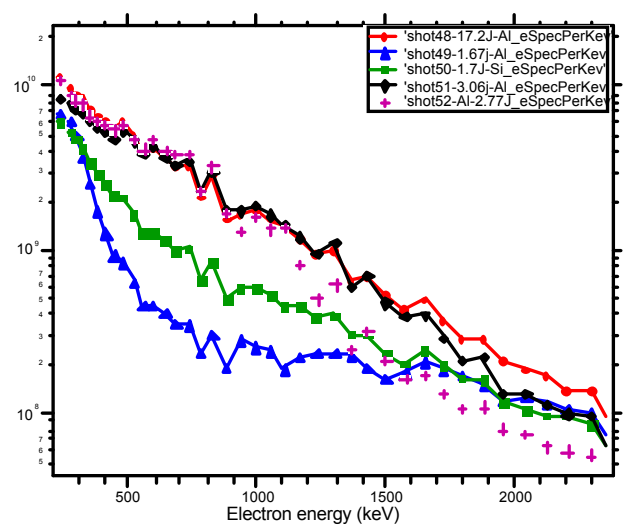

Figure 2. Electron distributions measured at various laser intensity at Callisto laser. The lowest curve (blue) is for $5 \times 10^{18} \mathrm{~W} / \mathrm{cm}^{2}$; The second lowest (green) is for $2 \times 10^{19} \mathrm{~W} / \mathrm{cm}^{2}$; The top three are for $6 \times 10^{19} \mathrm{~W} / \mathrm{cm}^{2}(\mathrm{red} \mathrm{cross})$; $8 \times 10^{19} \mathrm{~W} / \mathrm{cm}^{2}$ (black diamonds) and $3 \times 10^{20} \mathrm{~W} / \mathrm{cm}^{2}$ (red dots).

Similar observations were made at the RAL Vulcan Nd:glass Petawatt laser. This laser delivers over $400 \mathrm{~J}$ of energy onto target in a $400 \mathrm{fs}$ FWHM (full-width at half-maximum) temporal pulse onto a focu spot of about 7-8 $\mu \mathrm{m}$ [4]. The p-polarized laser was incident with at an angle of $28^{\circ}$ to the target normal. The peak intensity reaches up to $5 \times 10^{20} \mathrm{~W} / \mathrm{cm}^{2}$. Our electron spectrometer slit was aligned 15 degrees from the laser beam, i.e. 43 degrees off normal, and $79 \mathrm{~cm}$ away from the target. For the same target configuration (Ag target, $50 \mathrm{~mm}$ in thickness), we observed drastic changes in the hot electron distribution as a function of laser intensities, as illustrated in Fig. 3. However, at the same intensity, the electron distribution does not vary much for targets made of elements from $\mathrm{Al}$ to $\mathrm{Ag}$, a factor of 6 different in nuclear charge $\mathrm{Z}$.

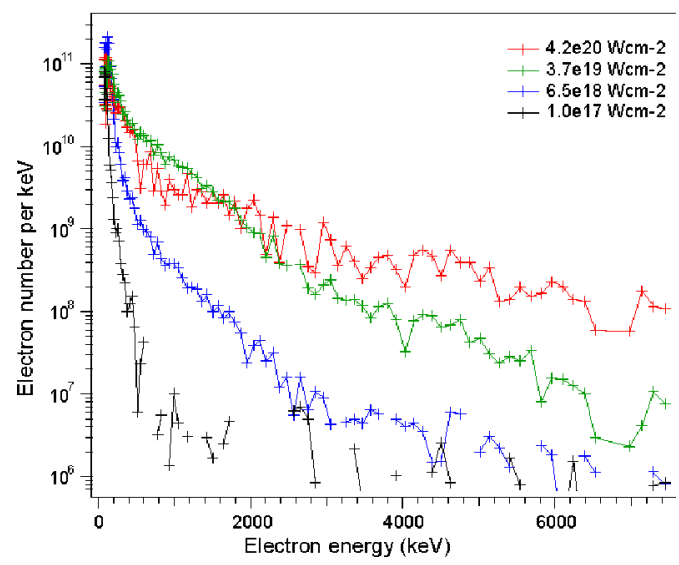

Figure 3. Electron distributions for $50 \mathrm{~mm} \mathrm{Ag}$ targets at four laser intensities. The hot electron temperatures are $0.1 \mathrm{MeV}, 0.4 \mathrm{MeV}, 0.7 \mathrm{Mev}$ and $1.6 \mathrm{MeV}$, respectively, for the four intensities from low to high order.

We plotted the experimental data from both LLNL Callisto and RAL Vulcan laser, in Fig. 4, along side the PIC simulation and ponderomotive scaling. We find that at lower intensities, the experimental data agree with the modeling and theory well, but a discrepency develops at higher intensities $\left(>10^{19}\right.$ 
$\mathrm{Wcm}^{-2}$ ), with the experimental temperatures being less than the predictions. A fit of the experimental data gives

$$
k T_{h o t} \sim\left(I \lambda^{2}\right)^{0.4}
$$

Note that this scaling is somewhere between the ponderomotive scaling (Eq.1) and scaling presented by Beg et. al. [6], which indicated that $k T_{h o t} \sim\left(I \lambda^{2}\right)^{0.3}$.

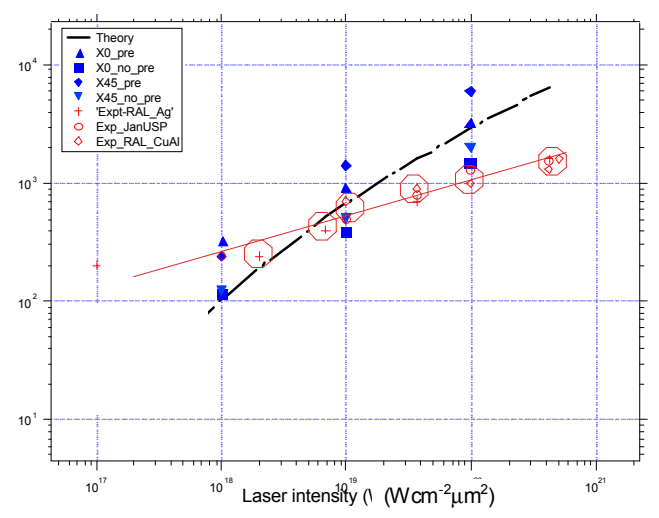

Figure 4. Experimental data (red crosses enclosed in circles) comparing to theoretical scaling (black dashed line) and 2D PIC modeling (as in Figure 1). The red solid line is the fit through the experimental data.

The physics behind this discrepancy between experimental and modeling results could lie in the interaction between short intense laser pulse and solid density plasma. As it has been pointed out earlier [7], for very high laser intensities, the electron density at the interaction surface increases considerably due to self-steepening as the laser pressure far exceeds the material pressure at the critical surface. As the density at this interface increases, the electrons that are generated there are less energetic. This results in lower hot electron temperatures, because the shorter the skin depth of the plasma due to the increase in electron density allows for less penetration of the laser's electronmagnetic field, thus producing less electron acceleration.

\section{SUMMARY}

We presented experimental measurement of hot electrons at laser intensity up to $5 \times 10^{20} \mathrm{~W} / \mathrm{cm}^{2}$. The fit to the experimental data reveal a scaling of hot electron temperature propotional to 0.4 power of the laser intensity.

\section{Acknowledgments}

This work was performed under the auspices of the U.S. Department of Energy by the University of California, Lawrence Livermore National Laboratory, under contract W-7405-ENG-48. The authors wish to thank the staff of the Central Laser Facility, CCLRC Rutherford Appleton Laboratory. 


\section{References}

[1] P. Gibbon and E. Forster, Plasma Phys. Control. Fusion, 38, 769 (1996).

[2] S. C. Wilks, W. L. Kruer, M. Tabak, and A. B. Langdon, Phys. Rev. Lett. 69, 1383 (1992).

[3] H. Chen, P. K. Patel, D. F.Price, B. K. Young, P. T. Springer, R. Berry, R. Booth, C. Bruns, and D. Nelson, Review Scientific Instruments, 74, 1551, (2003).

[4] P K Patel, M H Key, A J Mackinnon' et al. the Fast Ignitor Workshop Proceedings

[5] H. Chen, H. McLean, P. K. Patel, and S. C.Wilks, IFSA 03 (2003).

[6] Beg, F.N., Bell, A. R., Dangoor, A. E., Danson, C. N., Fews, A. P., Glinsky, M. E., Hammel, B. A., Lee, P., Norreys, P. A., and Tatarakis, M. Phys. Plasmas, 4, 447, (1997)

[7] S. C. Wilks and W. L. Kruer, IEEE J. of Quantum Electronics, 11, 1954 (1997). 